\title{
A Importância dos Testes Epicutâneos nos Episódios de DRESS: Estudo Comparativo 10 Anos Depois
}

\author{
Francisca Jácome Morgado', Luís Santiago', Margarida Gonçalo',2 \\ 'Serviço de Dermatologia, Centro Hospitalar e Universitário de Coimbra, Coimbra, Portugal \\ ${ }^{2}$ Faculdade de Medicina, Universidade de Coimbra, Coimbra, Portugal
}

\begin{abstract}
RESUMO - Introdução: A reação adversa a fármacos com eosinofilia e sintomas sistémicos (DRESS) é uma reação medicamentosa cutânea grave. Dependendo do(s) fármaco(s) envolvido(s), a sua imputabilidade pode ser confirmada por testes epicutâneos (TE). O objetivo foi avaliar o papel dos TE nos DRESS nos últimos 10 anos e comparar com o estudo prévio, 10 anos antes. Material e Métodos: Entre o período de 2009-2018 os doentes com o diagnóstico de DRESS realizaram TE no Serviço de Dermatologia do Centro Hospitalar e Universitário de Coimbra. Foram testados os principais fármacos imputáveis, assim como os iniciados posteriormente, e suspeitos de desencadear um agravamento do quadro. Os princípios ativos foram testados $1 \%-10 \%$ vas (Chemotechnique diagnostics ${ }^{\circledR}$ ), ou a partir de uma preparação comercial diluída a $10 \%$ vas. Resultados: O estudo incluiu 41 pacientes (20 homens, 21 mulheres, idade média 53 anos). Os principais fármacos envolvidos foram: alopurinol $(n=15)$, antiepiléticos $(n=14)$, sulfametoxazole-trimetoprim $(n=4)$, salazopirina $(n=3)$, diclofenac $(n=2)$ e antirretrovirais, ezetimibe-sinvastatina e ranelato de estrôncio, 1 cada. Em 15 doentes, outros fármacos $(n=18)$ foram suspeitos de agravar o episódio de DRESS: amoxicilina $(n=8)$, ciprofloxacina $(n=2)$, cefoxitina $(n=2)$ e levofloxacina, ceftriaxone, ceftazidime, vancomicina, aciclovir e metamizol ( 1 de cada). Obteve-se uma positividade ao fármaco principal em 10 doentes $(24,3 \%)$, todas relacionadas com antiepiléticos. Todos os doentes testados para o alopurinol obtiveram resultados negativos. A positividade para os fármacos associados a agravamento clínico foi de $67 \%$ (12/18), sem nenhuma positividade para as quinolonas. Conclusão: Globalmente os resultados foram similares aos obtidos no estudo anterior, no qual 18/56 (32,1\%) doentes obtiveram um resultado positivo no $T E$, com resposta positiva maioritariamente à carbamazepina e outros anticonvulsivantes. Diferentemente do estudo prévio, onde não foram testados fármacos administrados após o início da toxidermia, mostramos que os TE têm um papel importante no diagnóstico da cossensibilização, reforçando a importância de testar todos os medicamentos tomados durante o episódio. O reconhecimento de uma cossensibilização pode prevenir um novo DRESS desencadeada por um fármaco secundário.

PALAVRAS-CHAVE - Eosinofilia/diagnóstico; Exanthema/diagnóstico; Hipersensibilidade a Fármacos/diagnóstico; Testes Epicutâneos.
\end{abstract}

\section{The Importance of Patch Testing in Drug Reaction with Eosinophilia and Systemic Symptoms: 10 Years After}

ABSTRACT - Introduction: : Drug reaction with eosinophilia and systemic symptoms (DRESS) is a severe cutaneous adverse drug reaction. According on the culprit drug(s), imputability can be confirmed by patch test (PT). Our objective was to evaluate the value of PT in DRESS in the last 10 years, in comparison with our study in the preceding 10 years. Material \& Methods: From 2009 to 2018, patients with DRESS performed PT at the Dermatology Department of Coimbra University Hospital, with the main culprit drug(s) and all drugs administered concomitantly or suspected of inducing DRESS flares. Drugs at $1 \%-10 \%$ pet (Chemotechnique diagnostics ${ }^{\circledR}$ ) or from a commercial preparation diluted at 10\% pet were patch tested. Results: We studied 41 patients (20 male/ 21 female, mean age 53 years). The main culprits were allopurinol $(n=15)$, antiepileptics $(n=14)$, trimethoprim/ sulfamethoxazole $(n=4)$, salazopyrine $(n=3)$, diclofenac $(n=2)$, antiretrovirals, ezetimibe/simvastatin and strontium ranelate (1 each). In 15 patients other drugs $(n=18)$ were suspected of worsening DRESS, amoxicillin $(n=8)$, ciprofloxacin $(n=2)$, cefoxitin $(n=2)$, levofloxacin, ceftriaxone, ceftazidime, vancomycin, acyclovir and metamizole (1 each). A positive PT to the culprit drug

Correspondência: Francisca Jácome Morgado Serviço de Dermatologia

Centro Hospitalar e Universitário de Coimbra

Praceta, R. Prof. Mota Pinto

3004-561 Coimbra, Portugal

E-mail: fjacomemorgado@gmail.com

DOI: https://dx.doi.org/10.29021/spdv.78.1.1161
Recebido/Received 2020/01/27

Aceite/Accepted 2020/02/25

Publicado/Published 2020/04/--

(c) Autor (es) (ou seu (s) empregador (es)) e Revista SPDV 2020. Reutilização permitida de acordo com CC BY-NC. Nenhuma reutilização comercial.

(c) Author(s) (or their employer(s)) and SPDV Journal 2020. Re-use permitted under CC BY-NC. No commercial re-use. 


\section{Artigo Original}

was observed in 10 patients (24.3\%), all to antiepileptics. All patients tested for alopurinol had negative results. Positive reactions were observed to drugs related with flares in 12/18 suspected drugs (67\%), but not to quinolones. Conclusion: Results were similar to the study conducted 10 years before when 18/56 (32.1\%) patients had positive PT, mostly to carbamazepine and other anticonvulsants. Distinct from our previous study, when no tests were performed with the antibiotic series or other drugs used after the initiation of DRESS, we showed that PT can be a valuable tool to diagnose co-sensitisation in DRESS and emphasise the importance of testing all medications taken during the whole episode, even when PT has no value for the main culprit, like allopurinol. Recognising a co-sensitisation can prevent a new DRESS induced by the second drug.

KEYWORDS - Drug Hypersensitivity/diagnosis; Eosinophilia/diagnosis; Exanthema/diagnosis; Patch Tests.

\section{INTRODUÇÃO}

A reação adversa a fármacos com eosinofilia e sintomas sistémicos (DRESS ou síndrome de hipersensibilidade a fármacos) é uma reação cutânea grave com envolvimento multiorgânico que resulta de uma relação complexa entre fármacos e vírus num indivíduo suscetível. ${ }^{1}$ Os fármacos mais frequentemente envolvidos são: antiepiléticos, sulfonamidas, alopurinol, minociclina e antirretrovirais. ${ }^{2}$

Dada a sintomatologia associada ao pródromo inicial, frequentemente são tomados outros fármacos, nomeadamente antibióticos, ${ }^{3}$ podendo ocorrer uma cossensibilização associada a agravamento clínico. ${ }^{4}$

Para a avaliação da imputabilidade de um fármaco suspeito nos DRESS, os testes epicutâneos (TE) são a ferramenta mais útil. ${ }^{5}$ Estes mostraram ser um exame complementar seguro, com sensibilidade dependente do fármaco envolvido. ${ }^{6}$ Relativamente a outras alternativas, a prova de provocação oral não é aconselhada, uma vez que tem o potencial de desencadear uma reação adversa grave com risco de vida, e o teste de ativação linfocitária apresenta baixas sensibilidade e especificidade, não estando padronizado para a maioria dos fármacos. ${ }^{7}$

Em 2009, os autores publicaram um estudo que avaliou a utilidade dos TE nos DRESS ocorridos num centro hospitalar terciário, Centro Hospital e Universitário de Coimbra, num período de 10 anos (janeiro 1998 a dezembro 2008). ${ }^{8}$ É o objetivo deste trabalho avaliar retrospetivamente os resultados dos TE realizados em doentes com o diagnóstico de DRESS no mesmo centro hospitalar nos últimos 10 anos, e compará-los com o estudo anterior.

\section{MATERIAL E MÉTODOS}

\section{Seleção de pacientes e caracterização}

Foram incluídos os doentes com o diagnóstico de DRESS (casos possíveis, prováveis ou definitivos de acordo com os critérios RegiSCAR), ${ }^{9}$ que foram submetidos a TE no Centro Hospitalar e Universitário de Coimbra, no período de 1 de janeiro de 2009 a 31 de dezembro 2018. Os dados foram avaliados de uma forma retrospetiva.

\section{Testes Epicutâneos}

Os TE foram realizados 6 semanas a 6 meses após a resolução completa das lesões cutâneas, e pelo menos 1 mês após descontinuação dos corticóides sistémicos, de acordo com as normas de orientação da Sociedade Europeia de
Dermatites de Contacto. ${ }^{10}$ Todos os pacientes assinaram consentimento informado.

Os doentes foram testados com as séries europeias básica de alergénios de contacto, com os fármacos suspeitos e com a série correspondente dos fármacos química ou farmacologicamente relacionados (série de antiepiléticos, antibióticos, anti-inflamatórios não esteroides - AINES), com os fármacos introduzidos nas semanas antes, e em casos em que houve suspeita de reativação da toxidermia (flare), com todos os fármacos introduzidos após o início do DRESS.

Os testes foram realizados com os fármacos diluídos a $1 \%-10 \%$ em vaselina (Chemotechnique diagnostics ${ }^{\circledR}$ ) ou com a sua preparação comercial diluída de forma a ter o produto ativo a $10 \%$ vas ou com o pó do comprimido inclúdo a $30 \%$ vas. Os alergénios em finn chambers (Epitest Ld) ou IQUltra chambers (Chemotechnique diagnostics ${ }^{\circledR}$ ) foram aplicados no dorso e mantidos em oclusão durante 2 dias. As leituras foram realizadas em D3 ou D4 e com indicação para retomar ao centro hospitalar para uma nova leitura, caso surgisse alguma alteração a D7. Apenas reacções $1+$ ou mais intensas foram consideradas positivas.

\section{RESULTADOS}

\section{Características da população}

Foram incluídos 41 pacientes (20 homens e 21 mulheres) com uma idade média de $53 \pm 21,6$ anos (8-89). De acordo com os critérios RegiSCAR, os doentes foram categorizados como casos possíveis $(n=5)$, prováveis $(n=21)$ e definitivos $(n=15)$. Uma vez que não foram realizadas biópsias cutâneas ou estudo autoimune numa percentagem significativa dos doentes, a maioria dos casos foram apenas classificados como prováveis, não cumprido critérios suficientes para um diagnóstico definitivo.

As manifestações cutâneas desenvolveram-se numa média de $27 \pm 15$,8 dias (4-63) após a administração do fármaco e traduziram-se na maioria dos casos (81\%) sob a forma de um exantema maculopapuloso com envolvimento de $>50 \%$ da superfície corporal, seguindo-se de eritrodermia exfoliativa (19\%). Ocorreu edema facial em 85\% dos doentes. As manifestações sistémicas incluíram: febre $(88 \%)$, adenopatias $(41 \%)$, disfunção hepática $(82 \%)$, disfunção renal (26\%) e sintomatologia cardiorrespiratória (dispneia, tosse) (3\%). Dos doentes, 58\% apresentava eosinofilia no sangue periférico, tendo sido detetados linfócitos atípicos circulantes em cinco casos. Em todos os doentes 


\section{Artigo Original}

houve melhoria clínica com a suspensão do(s) fármaco(s) suspeito(s) de induzir ou agravar o DRESS e corticoterapia sistémica, com tempo médio para resolução das lesões cutâneas superior a 15 dias.

\section{Fármaco principal e resultados nos TE}

Tendo como base os achados clínicos e cronológicos o principal agente imputável foi o alopurinol ( $n=15,37 \%)$, seguido dos antiepiléticos $(n=14,34 \%)$. Os outros fármacos envolvidos foram: trimetropim/sulfametoxazole $(n=4)$, salazopirina $(n=3)$, diclofenac $(n=2)$, e antirretrovirais, ezetimibe/sinvastatina e ranelato de estrôncio (1 cada) (Tabela 1).

Obteve-se um resultado positivo nos TE em 10 doentes $(24,4 \%)$, sendo que todas as positividades corresponderam a fármacos do grupo dos antiepiléticos. Neste grupo, 9/10 das positividades foram à carbamazepina e 1 à fenitoína. A sensibilidade para a positividade à carbamazepina foi de 100\% (Tabela 2).

Não se obteve nenhum resultado positivo para o alopurinol, o principal fármaco envolvido.

Tabela 1 - Fármacos principais e resultados nos TE.

\begin{tabular}{l|c|c|c}
\multicolumn{1}{c|}{ Fármaco } & Doentes (N) & $\begin{array}{c}\text { TE } \\
\text { Positivo (N) }\end{array}$ & $\begin{array}{c}\text { TE } \\
\text { Positivo (\%) }\end{array}$ \\
\hline Alopurinol & 15 & 0 & 0 \\
\hline Antiepilépticos & 14 & 10 & 71,4 \\
\hline $\begin{array}{l}\text { Trimetropim / } \\
\text { Sulfametoxazole }\end{array}$ & 4 & 0 & 0 \\
\hline Salazopirina & 3 & 0 & 0 \\
\hline Diclofenac & 2 & 0 & 0 \\
\hline Antirretrovirais & 1 & 0 & 0 \\
\hline $\begin{array}{l}\text { Ezetemibe/ } \\
\text { Sinvastatina }\end{array}$ & 1 & 0 & 0 \\
\hline $\begin{array}{l}\text { Ranelato de } \\
\text { estrôncio }\end{array}$ & 1 & 0 & 0 \\
\hline Total & 41 & 10 & $\mathbf{2 4 , 4}$ \\
\hline
\end{tabular}

Tabela 2 - Antiepilépticos e resultados nos TE.

\begin{tabular}{l|c|c|c}
\multicolumn{1}{c|}{ Antiepilético } & Doentes (N) & $\begin{array}{c}\text { TE } \\
\text { Positivo (N) }\end{array}$ & $\begin{array}{c}\text { TE } \\
\text { Positivo (\%) }\end{array}$ \\
\hline Carbamazepina & 9 & 9 & 100 \\
\hline Fenitoína & 3 & 1 & 33 \\
\hline Eslicarbazepina & 1 & 0 & 0 \\
\hline Ácido valpróico & 1 & 0 & 0 \\
\hline Total & 14 & 10 & 71,4 \\
\hline
\end{tabular}

Em nenhum dos casos houve reativação das lesões cutâneas ou surgimento de sintomas sistémicos durante ou após a realização dos testes.

Todas as positividades foram obtidas em D3/D4, sem necessidade de leitura tardia adicional (D7).

\section{Fármacos associados a flare e resultados nos TE}

Em 15 doentes a toma de outros fármacos $(n=18)$ após o início do episódio de DRESS foi associada a uma reativação clínica do episódio de DRESS - flare. Destes, o fármaco mais frequentemente envolvido foi a amoxicilina $(n=8)$ seguido de ciprofloxacina $(n=2)$, cefoxitina $(n=2)$ e levofloxacina, ceftriaxone, ceftazidime, vancomicina, aciclovir e metamizol (1 cada) (Tabela 3).

Tabela 3 - Fármacos associados a flare e resultados nos TE.

\begin{tabular}{l|c|c|c}
\multicolumn{1}{c|}{ Fármaco } & Doentes (N) & $\begin{array}{c}\text { TE } \\
\text { Positivo (N) }\end{array}$ & $\begin{array}{c}\text { TE } \\
\text { Positivo (\%) }\end{array}$ \\
\hline Amoxicilina & 8 & 6 & 75 \\
\hline $\begin{array}{l}\text { Cefalosporinas } \\
\text { Ceftazidime, } \\
\text { Cefoxitina, } \\
\text { Ceftriaxona }\end{array}$ & 3 & 3 & 100 \\
\hline Vancomicina & 1 & 1 & 100 \\
\hline Aciclovir & 1 & 1 & 100 \\
\hline Metamizol & 3 & 0 & 100 \\
\hline $\begin{array}{l}\text { Quinolonas } \\
\text { Ciprofloxacina, } \\
\text { Levofloxacina }\end{array}$ & 18 & 12 & 67 \\
\hline Total & 1 & 1 & 0 \\
\hline
\end{tabular}

Relativamente aos TE, foram obtidos resultados positivos em $67 \%$ dos casos (12/18): amoxicilina $(n=6)$, cefalosporinas $(n=3)$, vancomicina $(n=1)$, aciclovir $(n=1)$ e metamizol $(n=1)$. Não se obteve nenhuma positividade às quinolonas. Excluindo as quinolonas, a taxa de resposta positiva para os fármacos associados com flare foi de $80 \%$.

Todas as positividades foram obtidas em D3/D4, sem necessidade de leitura tardia adicional (D7).

\section{DISCUSSÃO}

Os resultados do estudo foram similares aos resultados obtidos 10 anos antes, quando 32,1\% dos doentes (18/56) obtiveram resultados positivos nos TE. A positividade foi inferior neste estudo $(24,4 \%)$, provavelmente devido ao uso de novos antiepilépticos mais seguros em prol dos antiepilépticos aromáticos convencionais associados a mais reações adversas $^{11}$ (no período 1998-2008 os antiepilépticos eram o fármaco suspeito em 33 doentes vs 14 em 2009-2018).

Como esperado, a sensibilidade dos TE foi altamente dependente dos fármacos envolvidos, o que pode ser 


\section{Artigo Original}

ilustrado pelos resultados divergentes dos dois principais grupos farmacológicos: $71,4 \%$ de resposta positiva aos antiepilépticos e ausência de reatividade ao alopurinol (também no estudo anterior sem positividades). Embora já concluído anteriormente que os TE não permitem o diagnóstico de DRESS induzido por alopurinol, continuámos a testar este subgrupo de doentes com o intuito de diagnosticar possíveis cossensibilizações.

Relativamente aos mecanismos associados à ausência de resposta ao alopurinol, não existindo resposta definitiva, são especuladas várias hipóteses:

1) o agente responsável final é um metabolito do fármaco que não se forma durante o TE;

2) não existe mecanismo imunológico envolvido;

3) estão envolvidos outros fatores numa intolerância ao fármaco transitória, como infeções virais, que não estão presentes na altura do TE;

4) administração incorreta do fármaco, com concentração e tempos de exposição inadequados, como nos indivíduos com patologia renal concomitante. ${ }^{12}$

No grupo dos antiepiléticos, o principal fármaco envolvido foi a carbamazepina, principal antiepilético associado a episódios de DRESS, com uma positividade de $100 \%$, concordante com a literatura. ${ }^{13,14}$

Os antibióticos são frequentemente administrados durante o episódio de DRESS devido à sintomatologia inicial. A exposição a fármacos não relacionados pode induzir uma neossensibilização associada a reativação clínica ou mesmo um novo DRESS durante o rechallenge com o segundo fármaco. ${ }^{15} \mathrm{~A}$ hipersensibilidade a múltiplos fármacos pode ocorrer em até $50 \%$ dos pacientes com DRESS. ${ }^{16}$ O mecanismo sugerido é que ocorra uma ativação massiva do sistema imunitário em que o novo fármaco interage $\mathrm{com}$ as células $T$ pré-ativadas com afinidade suficiente para desencadear uma ativação e expansão destas células, ocorrendo uma nova sensibilização. ${ }^{17} \mathrm{~A}$ reprodutibilidade dos TE aos antibióticos após vários anos suporta a força desta reação mediada por células T. ${ }^{18}$

Assim, em acréscimo ao estudo anterior, foram testados outros fármacos, na sua maioria antibióticos, administrados após o episódio do DRESS e associados com flares. Neste grupo de pacientes, os TE revelaram-se especialmente importantes, com uma elevada positividade (67\%), mesmo quando não houve resposta ao fármaco principal.

A positividade dos TE com um fármaco secundário permite o diagnóstico de uma cossensibilização e o seu reconhecimento poderá prevenir um novo DRESS induzido por um fármaco diferente do imputável ao episódio inicial. Assim realça-se a necessidade de testar todos os fármacos tomados antes e durante todo o episódio de DRESS. Para prevenir uma neossensibilização deve ser evitada a introdução de novos fármacos, e em caso de necessidade, deve ser protelada (de forma a que o sistema imunitário não esteja na sua fase inicial hiperativa) ou introduzida com corticoterapia oral associada. ${ }^{17}$

\section{CONCLUSÃO}

Os TE são seguros nos DRESS, sendo particularmente úteis em casos de hipersensibilidade aos antiepilépticos, sobretudo carbamazepina. Os TE não têm valor para detectar a imputabilidade associada ao alopurinol. No entanto, mesmo quando não têm utilidade para o fármaco principal, estes são úteis para o diagnóstico de uma cossensibilização. Assim, é importante testar todos os fármacos/antibióticos tomados durante o episódio, para o seu reconhecimento e consequente prevenção de uma nova reação medicamentosa.

Conflitos de interesse: Os autores declaram a inexistência de conflitos de interesse na realização do presente trabalho.

Fontes de financiamento: Não existiram fontes externas de financiamento para a realização deste artigo.

Confidencialidade dos dados: Os autores declaram ter seguido os protocolos da sua instituição acerca da publicação dos dados de doentes.

Protecção de pessoas e animais: Os autores declaram que os procedimentos seguidos estavam de acordo com os regulamentos estabelecidos pelos responsáveis da Comissão de lnvestigação Clínica e Ética e de acordo com a Declaração de Helsínquia da Associação Médica Mundial.

Proveniência e revisão por pares: Não comissionado; revisão externa por pares.

Conflicts of interest: The authors have no conflicts of interest to declare.

Financing support: This work has not received any contribution, grant or scholarship.

Confidentiality of data: The authors declare that they have followed the protocols of their work center on the publication of data from patients.

Protection of human and animal subjects: The authors declare that the procedures followed were in accordance with the regulations of the relevant clinical research ethics committee and with those of the Code of Ethics of the World Medical Association (Declaration of Helsinki).

Provenance and peer review: Not commissioned; externally peer reviewed

\section{ORCID}

Margarida Gonçalo

http://orcid.org/0000-0001-6842-1360

Francisca Jácome Morgado

http://orcid.org/0000-0001-5006-4334

Luís Santiago

http://orcid.org/0000-0003-2587-5535

\section{REFERÊNCIAS}

1. Husain Z, Reddy BY, Schwartz RA. DRESS syndrome: Part I. Clinical perspectives. J Am Acad Dermatol. 2013; 68:693.e1-14; quiz 706-8. doi: 10.1016/j. jaad.2013.01.033.

2. Shiohara T, Mizukawa Y. Drug-induced hypersensitivity syndrome (DiHS)/drug reaction with eosinophilia and systemic symptoms (DRESS): An update in 2019. Allergol 


\section{Artigo Original}

Int. 2019; 68:301-8. doi: 10.1016/j.alit.2019.03.006.

3. Mardivirin L, Valeyrie-Allanore L, Branlant-Redon E, Beneton N, Jidar K, Barbaud A, et al. Amoxicillin-induced flare in patients with DRESS (Drug Reaction with Eosinophilia and Systemic Symptoms): report of seven cases and demonstration of a direct effect of amoxicillin on Human Herpesvirus 6 replication in vitro. Eur J Dermatol. 2010; 20:68-73. doi: 10.1684/ejd.2010.0821.

4. Pichler WJ, Srinoulprasert Y, Yun J, Hausmann O. Multiple Drug Hypersensitivity. Int Arch Allergy Immunol. 2017; 172:129-38. doi: 10.1159/000458725.

5. Barbaud A, Collet E, Milpied B, Assier H, Staumont D, Avenel-Audran $M$, et al. A multicentre study to determine the value and safety of drug patch tests for the three main classes of severe cutaneous adverse drug reactions. Br J Dermatol. 2013; 168:555-62. doi: 10.1111/ bjd. 12125.

6. Pinho A, Coutinho I, Gameiro A, Gouveia M, Gonçalo $M$. Patch testing - a valuable tool for investigating non-immediate cutaneous adverse drug reactions to antibiotics. J Eur Acad Dermatol Venereol. 2017; 31 : 280-7. doi: $10.1111 /$ jdv. 13796 .

7. Soria A, Hamelin A, de Risi Pugliese T, Amsler E, Barbaud $A$. Are drug intradermal tests dangerous to explore cross-reactivity and co-sensitization in DRESS? Br J Dermatol. 2019; 181:611-2. doi: 10.1111/bjd.17835.

8. Santiago F, Gonçalo M, Vieira R, Coelho S, Figueiredo A. Epicutaneous patch testing in drug hypersensitivity syndrome (DRESS). Contact Dermatitis. 2010; 62:4753. doi: 10.1111/ן.1600-0536.2009.01659.x.

9. Kardaun SH, Sidoroff A, Valeyrie-Allanore L, Halevy S, Davidovici BB, Mockenhaupt M, et al. Variability in the clinical pattern of cutaneous side-effects of drugs with systemic symptoms: does a DRESS syndrome really exist? $\mathrm{Br}$ J Dermatol. 2007; 156:609-11. doi: 10.1111/i.1365$-2133.2006 .07704 . x$.

10. Johansen JD, Aalto-Korte K, Agner T, Andersen KE, Bircher A, Bruze M, et al. European Society of Contact
Dermatitis guideline for diagnostic patch testing - recommendations on best practice. Contact Dermatitis. 2015; 73:195-221. doi: 10.1111/cod.12432.

11. Błaszczyk B, Lason W, Czuczwar SJ. Antiepileptic drugs and adverse skin reactions: An update. Pharmacol Rep. 2015;67:426-34. doi: 10.1016/i.pharep.2014.11.009.

12. Vieira $R$, Gonçalo $M$, Figueiredo $A$. Testes epicutâneos ao alopurinol e oxipurinol em doentes com toxidermias ao alopurinol. Trab Soc Port Dermatol Venereol. 2004;62:247-53.

13. Özkaya E, Kılıç S. Severe adverse cutaneous drug reactions to antiepileptic drugs: 18 years of experience in a tertiary referral dermatology clinic in Turkey. J Eur Acad Dermatol Venereol. 2018; 32:e268-e270. doi: 10.1111/jdv. 14799.

14. Ben Mahmoud L, Bahloul N, Ghozzi H, Kammoun B, Hakim A, Sahnoun Z, et al. Place du patch-test dans le diagnostic des reactions d'hypersensibilites retardees induites par les antiepileptiques. Therapie. 2017; 72:53945. doi: 10.1016/i.therap.2017.01.011.

15. Batista M, Cardoso JC, Oliveira P, Gonçalo M. Allopurinol-induced DRESS syndrome presented as a cholecystitis-like acute abdomen and aggravated by antibiotics. BMJ Case Rep. 2018. doi: 10.1136/bcr-2018-226023.

16. Santiago LG, Morgado FJ, Baptista MS, Gonçalo M. Hypersensitivity to antibiotics in drug reaction with eosinophilia and systemic symptoms (DRESS) from other culprits. Contact Dermatitis. 2020(in press). doi: 10.1111/ cod. 13462.

17. Pichler WJ, Daubner B, Kawabata T. Drug hypersensitivity: flare-up reactions, cross-reactivity and multiple drug hypersensitivity. J Dermatol. 2011; 38:216-21. doi: 10.1111/i.1346-8138.2010.01142.x.

18. Pinho A, Marta A, Coutinho I, Gonçalo M. Long-term reproducibility of positive patch test reactions in patients with non-immediate cutaneous adverse drug reactions to antibiotics. Contact Dermatitis. 2017; 76:204-9. doi: $10.1111 / \mathrm{cod} .12720$. 